\title{
Study on Arc Envelope Grinding Process of Revolving Curved Surface Lingye Kong ${ }^{1, a}$,Qiusheng Yan ${ }^{2, b}$,Yongliang Qiu $^{1, \mathrm{c}}$ and Tengxiong Qiu ${ }^{1, \mathrm{~d}}$ \\ ${ }^{1}$ GuangDong Polytechnic of Industry and Commerce, Guangzhou, China \\ ${ }^{2}$ Guangdong University of Technology, Guangzhou, China \\ akonglingye@yeah.net, ${ }^{\mathrm{b}}$ qsyan@gdut.edu.cn
}

Keywords: Arc grinding wheel, curved surface, roughness uniformity, variable speed machining

\begin{abstract}
To meet the precision machining needs of axisymmetric revolving curved surface, the arc envelope grinding technology for curved surface is studied so as to make a breakthrough in the current process. The particularity of arc envelope grinding of curved surface is analyzed. Through the analysis of the forming process of grinding curved surface, the geometric model of the grinding wheel interfering with the workpiece during the arc envelope grinding is established. Through the analysis of the moving distance of grinding point, the relationship between the effective moving distance, the radius of arc of grinding wheel, and the curvature radius of workpiece is established. It is optimized and analyzed that the grinding point on the surface of grinding wheel has the maximum motion quantity to improve the wear resistance of grinding wheel. Based on the calculation of curvature of machined curved surface, the change in feeding rate can be adjusted to ensure the uniform surface roughness of the curved surface. It provides a theoretical basis for the practical application of Arc Envelope Grinding Method (AEGM) for curved surface.
\end{abstract}

\section{Introduction}

The typical parts with axisymmetric revolving curved surface include the side plate of tire mold, mold core of luminaire, rolling wheel of rolled tube, etc. It is characterized by the shape accuracy and surface roughness of curved surface which has a decisive impact on product performance.

Currently, according to the requirements on material, shape and precision and other factors, the processing methods adopted for the axisymmetric revolving curved surface are different. For example, the ultra-precision single point diamond turning method can achieve the mirror forming and processing of curved surface via the ultra-precision machine tool, but it is only limited to the precision machining of copper, aluminum, nickel and other soft mold materials, and it is difficult for the precision machining of hardening steel, high speed steel, hard alloy and ceramic and other superhard mold materials. As for the optical glass, ceramic and other high-brittle materials difficult for processing, the precision grinding, lapping and polishing methods are mainly adopted to achieve processing[1]. Meanwhile, as for the axisymmetric curved surface with a small size, the compression molding method via mold, or directly the single point diamond turning method can be used[2]. For large curved surfaces, the combination process method is often used. For example, the CNC machining is used to meet the accuracy requirements, and then the lapping and polishing are adopted to meet the requirement of surface roughness.

To meet the precision machining need and the requirements of shape accuracy and surface accuracy of large axisymmetric revolving curved surface, the grinding process must be used. In this paper, based on the arc trajectory envelope method, the grinding method by using a thick grinding wheel with a large-diameter circular section is proposed[3]. The features of arc envelope grinding process of revolving curved surface are completely different from those of cylinders, planes and other surface. For example, the contact state of surface between the grinding wheel and the workpiece is changed, and the degree of overlapping between the surface of grinding wheel and the machined surface is significantly reduced, so the machining process has its own peculiarities, and many new problems still need systematic study. This paper mainly analyzes the differences between curved surface grinding, plane grinding and external grinding. Based on the envelope grinding method of arc 
grinding wheel, the theoretical analysis is made on the movement of the grinding point on the surface of grinding wheel. And the parameters of grinding process are optimized.

\section{Arc Envelope Grinding Method (AEGM) for Axisymmetric Revolving Curved Surface}

From Fig.1, we know that the axisymmetric revolving curved surface is formed by a curve (as generatrix) rotating around the fixed axis. The curved surface has the features of an axisymmetric structure. To achieve precise trajectory envelope grinding of revolving curved surface, the working surface of an ordinary grinding wheel is trued to arc revolving surface so as to avoid geometric interference between the grinding wheel and the workpiece[4]. The machining method has the biggest advantages: with the change of grinding position of curved surface, the grinding point on the arc surface of grinding wheel is moving; the grinding doesn't need the fixed point any longer, so more abrasive partices on the grinding wheel can participate in cutting, and the partial wear of grinding wheel can be reduced and the service life of the grinding wheel can be improved. On the other hand, the curve interpolation can be completed by controlling the two-axis linkage. So the motion control of grinding wheel during machining is simplified.
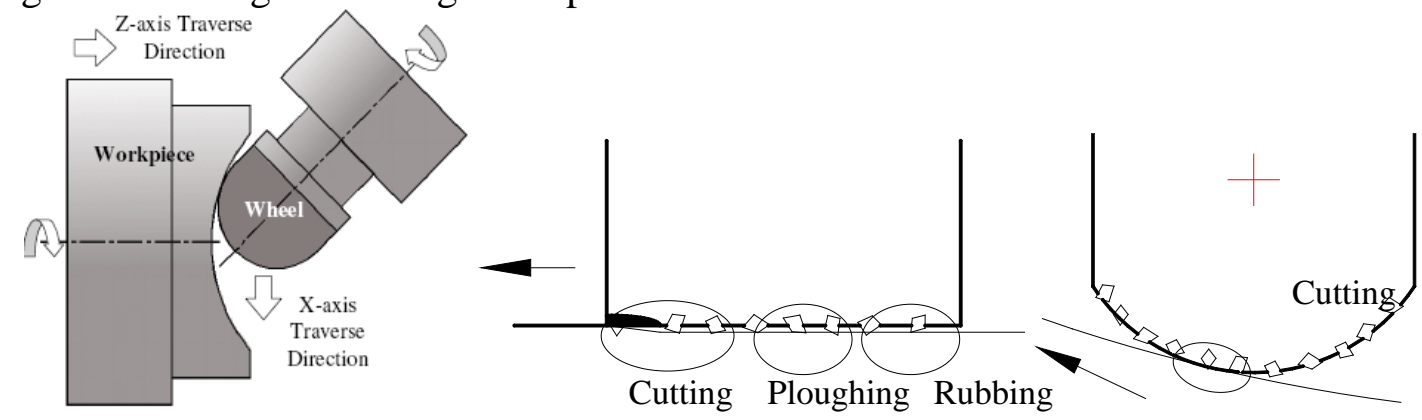

Fig.1 Arc envelope grinding method

Fig.2 Contact mode of envelope grinding method

Analysis of Arc Envelope Grinding Process of Curved Surface. For the AEGM, due to the presence of curvature of the revolving curved surface, the characteristics of envelope grinding process have great changes compared with those of cylinders, planes and other surface. For example, the contact area between the grinding wheel and the workpiece surface becomes smaller, and the overlapping between the surface of grinding wheel and workpiece is significantly reduced, so it shows different machining processes and control rules. It can be specificly summarized as follows: Contact mode

For plane grinding or external and internal grinding, the contact mode between the grinding wheel and the workpiece surface is surface or line contact. Some abrasive partices on the periphery of grinding wheel mainly involve in grinding. And the rest of abrasive partices are for buffing on the machined surface, mainly playing a role in rubbing and finishing. The formed grinding surface is the result of buffing by the grinding wheel for several times, so the grinding efficiency is high, and the roughness of machined surface is low.

For the arc envelope grinding of curved surface, the point contact always exists between the grinding wheel (with the arc revolving surface) and the curved surface at any position. When moving to the next grinding position, the abrasive partices which contacted with the surface of grinding wheel have been away from the workpiece. Therefore, the abrasive partices cannot continue the grinding, and the curved surface formed by grinding basically has no buffing process of plane grinding. Under the same process conditions, the material processed by arc envelope curve grinding has a low removal rate, and a high surface roughness, as shown in Fig. 2. Therefore, during the grinding of workpiece, the shape error and wear value of the grinding wheel are overwritten to the same position on the workpiece $^{[5]}$. From the point of view of the workpiece shape, the shape accuracy of the arc radius of the grinding wheel shall be ensured and the local wear shall be minimized. 


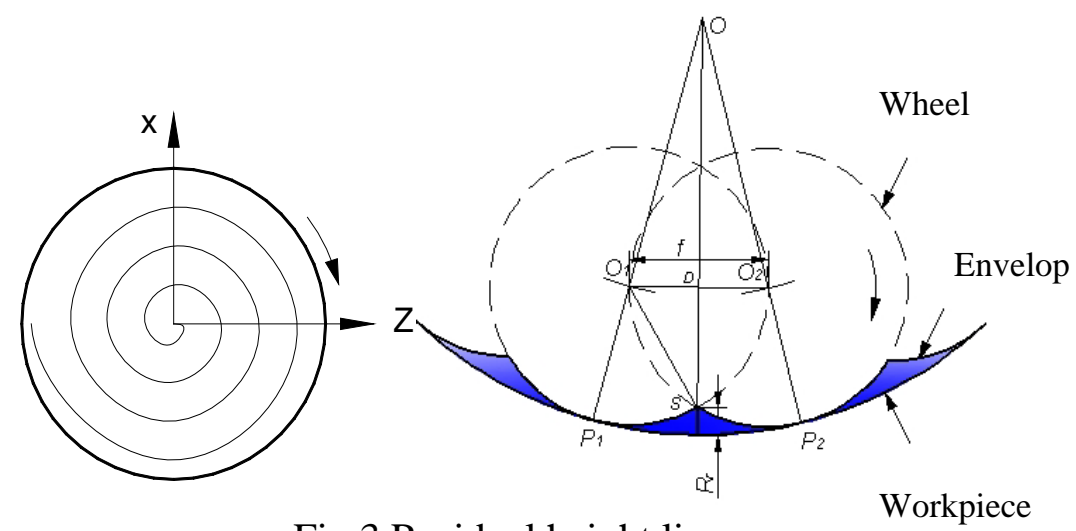

Fig.3 Residual height line

Theoretical Residual Height of Machined Surface.During the grinding of revolving curved surface, there is a residual height between the adjacent processing paths on the workpiece. The value of residual height depends on the rotational speed, feeding rate of the workpiece and other motion relationships.

Fig. 3 shows the calculation schematic of residual height between the adjacent trajectories of grinding wheel. P1 and P2 are the two points on the curved surface. When their adjacent spacing is small, their radius of curvature $\rho$ can be approximate to equal. Suppose that the center distance $f$ of adjacent processing trajectories on the grinding wheel is, and the arc radius $r$ of the wheel is. According to the geometric relationships in the figure, the residual height $R r$ is as follows ${ }^{[3]}$ :

$$
R_{r}=\rho-\sqrt{(\rho-r)^{2}-v_{r}^{2} /\left(4 n_{w}^{2}\right)}-\sqrt{r^{2}-v_{r}^{2} /\left(4 n_{w}^{2}\right)} .
$$

The radius of curvature in a different position of the workpiece is different, so the value of residual height is also different, resulting in uneven surface roughness on the curved surface of the entire workpiece. Controlling the step pitch and feeding rate of grinding wheel is a solution to keep the value consistent.

Movement of Grinding Point. During the envelope grinding of arc grinding wheel with curved surface, the point contact always exists between the surface of grinding wheeland the curved surface of the workpiece. In order to reduce wheel wear, the contact length of arc section can be increased to slow down the wheel wear and improve its shape retention, so as to ensure the shape accuracy of machined curved surface and maintain a stable grinding performance. Depending on the change condition of curvature of machined curved surface, the structural parameters of grinding wheel can be optimized to improve the effective utilization ratio of the abrasive partices, achieving uniform wear of the grinding wheel. First, it is necessary to study the effective grinding length of abrasive partice on the surface of grinding wheel.

Suppose that the generatrix of revolving curved surface is a parabola $\left(z=a y^{2}\right)$. When the point $\mathrm{A}$ moves to the point $\mathrm{B}$, the included angle between the connecting line (between the center of arc contour of the grinding wheel and the grinding point) and the vertical axis is $\theta$. Suppose that the curvature of the parabola at any point is $q$. The radius of curvature of any point on the revolving curved surface is $\rho_{n}$, and the distance between the grinding point and the center of grinding wheel is $R$, as shown in Fig. 4 .

According to the principle of differential geometry, from point A to point $\mathrm{B}$, the arc length $L$ participating in grinding on the periphery of grinding wheel is as follows:

$$
L=\int_{\theta 1}^{\theta 2} r \bullet d \theta=\int_{y 1}^{y 2} \frac{2 a r}{1+(2 a y)^{2}} d y=\int_{y 1}^{y 2} \frac{2 a r}{(2 a \rho)^{2 / 3}} d y=\frac{2 a r\left(y_{2}-y_{1}\right)}{(2 a \rho)^{2 / 3}}
$$




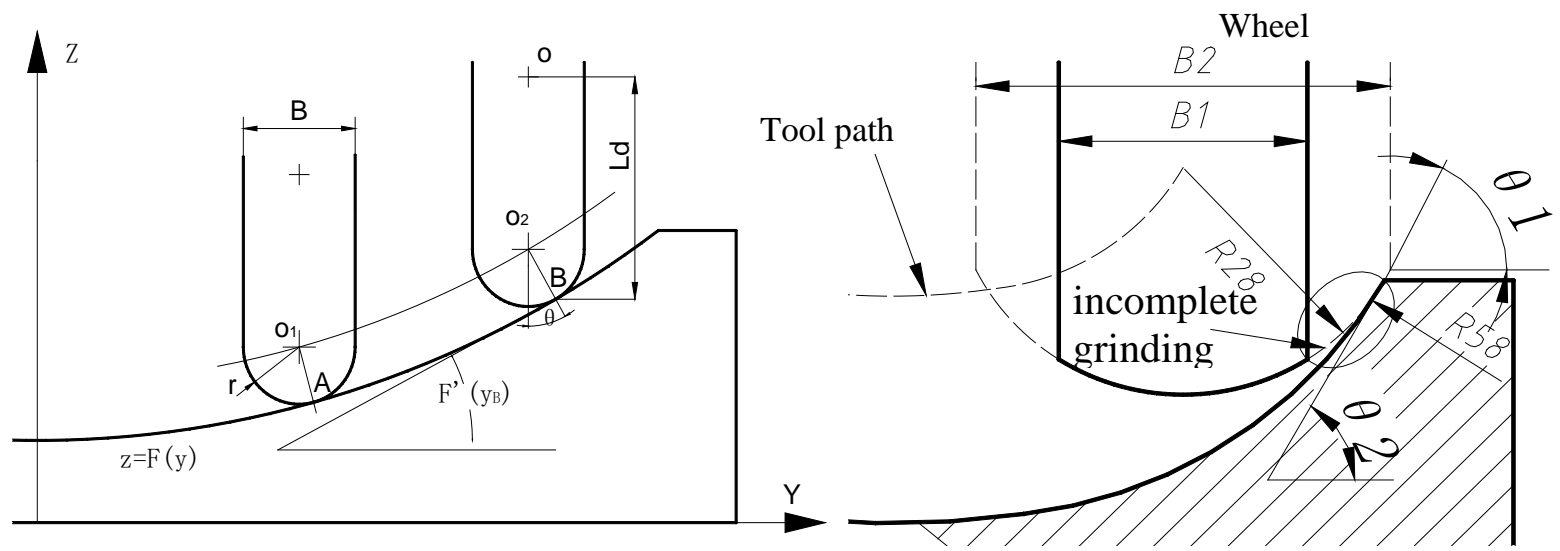

Fig. 4 Calculation of effective grinding width Fig.5 Schematic of incomplete grinding of workpiec

From the above equation, when the radius of curvature $\rho$ of the curved surface increases, the length of motion trajectory of grinding point decreases. Meanwhile, when the radius of curvature of workpiece is given, try to choose a large arc radius $r$ of curvature so as to increase the wear resistance of the grinding wheel.

However, for the grinding wheel with a given width, if the arc radius of wheel is too large, then the workpiece may not be completely ground, as shown in the circle in Fig. 5 . When the wheel width is $B 2$, and the arc center of wheel is moved to the offset position of the cutter (R58), then the curved surface can be completely ground. When the wheel width is $B 1$, the arc radius of wheel is also less than the minimum of two radiuses of curvature on the curved surface; since the width is small, the arc cross-section is short, it will lead to incomplete grinding of the curved surface. Therefore it is necessary to study the relationship between the wheel width and the arc radius.

As shown in Fig. 5, when the included angle $\theta_{1}$ between the tangent line (at the edge of arc of wheel cross-section) and the horizontal plane is larger than the included angle $\theta_{2}$ between the tangent line (at the point with the maximum slope on the entire curved surface of the workpiece) and the horizontal plane, the complete grinding can be achieved. Therefore, when $\theta 1 \geq \theta 2$, the incomplete grinding phenomenon does not occur.

While $\theta 2=\operatorname{arctg} F^{\prime}\left(y_{B}\right)$

According to the geometric relationship, $\sin \theta 1=\frac{B / 2}{r}=\frac{B}{2 r}$,

So, to ensure complete grinding, we have:

$$
\arcsin \frac{B}{2 r} \geq \operatorname{arctg} F^{\prime}\left(y_{B}\right)
$$

In summary, in the entire grinding process of the curved surface, the arc radius of grinding wheel must be as follows:

$$
\left\{\begin{array}{l}
r \leq \rho_{\min }=1 /|2 a| \\
\arcsin B / 2 r \geq \operatorname{arctg} F^{\prime}\left(y_{B}\right)
\end{array}\right.
$$

Contact Arc Length.For the grinding of revolving curved surface, due to the changes in surface curvature, from the microscopic point of view, the contact arc length and especially the contact area are also changed. When using AEGM, the changes in contact arc length cannot be ignored.If the inverse grinding method is used, then the contact arc length is ${ }^{[6,7]}$ :

$$
l_{s e}=\sqrt{\left(1+\frac{v_{w}}{v_{s}}\right)^{2}+\left(\frac{f_{a} n_{w}}{v_{s}}\right)^{2}} \sqrt{\frac{d_{s} d_{w} a_{p}}{d_{s}+d_{w}}}
$$

Where, $v_{s}$---- wheel speed $(\mathrm{m} / \mathrm{s}), v_{w}$---- workpiece speed $(\mathrm{m} / \mathrm{min}), f_{a}$---- axial feed rate $(\mathrm{mm} / \mathrm{r})$, $n_{w}$---- workpiece rotational speed $(\mathrm{r} / \mathrm{min}), d_{s}$---- wheel diameter $(\mathrm{mm}), d_{w}$---- workpiece diameter (mm), $a_{p}$---- grinding depth (mm) 
The position of the grinding point contacting with the surface of grinding wheel is variational, so the values $d_{s}, d_{w}, v_{s}, v_{w}$ in the formula are also variational, which means that the contact arc length is variational.

According to the structural parameters of roller die and the wheel size shown in Fig. 6, suppose that $n_{w}$ is $150 \mathrm{r} / \mathrm{min}, v_{s}$ is $35 \mathrm{~m} / \mathrm{s}$, wheel feeding rate $V r$ is $5 \mathrm{~mm} / \mathrm{min}$, and ap is $0.02 \mathrm{~mm}$. The changes in contact arc length lse between the grinding wheel and curved surface with the change of processing parameters are calculated and analyzed, as listed in Table 1. The contact arc lengths at different parts of the grinding wheel are shown in Fig. 7.

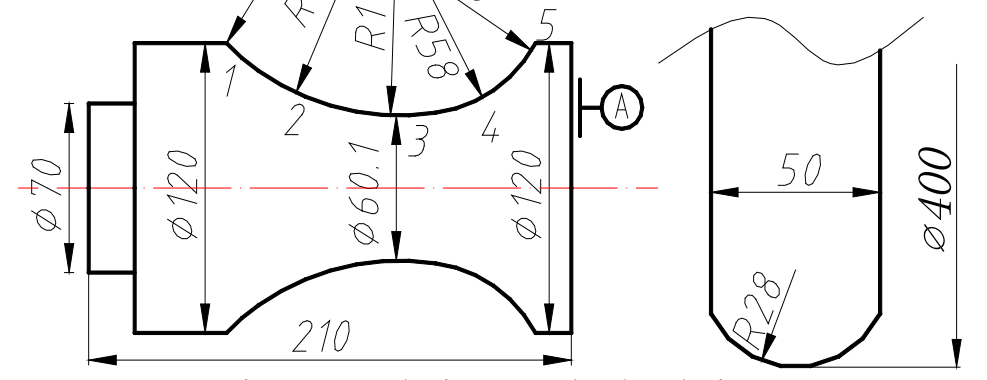

Fig.6 Workpiece and wheel size

Table1 Contact arc length at different positions

\begin{tabular}{cccccccc}
\hline${ }^{\mathrm{N}}$ & $V s(\mathrm{~m} / \mathrm{s})$ & $V w(\mathrm{~m} / \mathrm{s})$ & $F(\mathrm{~mm} / \mathrm{s})$ & $d s(\mathrm{~mm})$ & $d w(\mathrm{~mm})$ & $a p(\mathrm{~mm})$ & lse $(\mathrm{mm})$ \\
\hline 1 & 33.46 & 0.942 & 0.083 & 382.88 & 120 & 0.02 & 1.3899 \\
2 & 34.54 & 0.6206 & 0.083 & 395.2 & 79.06 & 0.02 & 1.1685 \\
3 & 34.96 & 0.4718 & 0.083 & 400 & 60.1 & 0.02 & 1.036 \\
4 & 34.385 & 0.5901 & 0.083 & 393.44 & 75.17 & 0.02 & 1.143 \\
5 & 32.49 & 0.942 & 0.083 & 371.8 & 120 & 0.02 & 1.386 \\
\hline
\end{tabular}
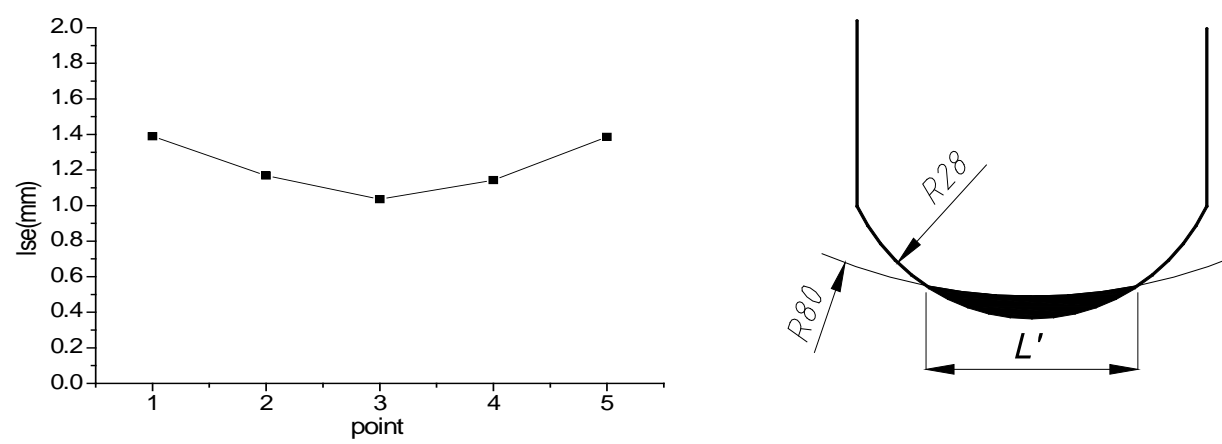

Fig.7 The value of contact arc length Fig.8 Envelope contact width between wheel and workpiece

Thus, the contact arc length differs due to the different parameters. According to the results in the table, during the grinding at the lowest point of the curved surface, the contact arc length between the grinding wheel and the workpiece is minimal and $25.5 \%$ smaller than the contact arc length on both ends. Furthermore, when the arc radius R28 of arc section of grinding wheel is certain and the cutting depth is $0.02 \mathrm{~mm}$, the envelope contact width $L^{\prime}$ is also related to the radius of curvature of the workpiece, as shown in Fig. 8. The values sorted by size are $L^{\prime} 58>L^{\prime} 80>L^{\prime} 110.4$. Therefore, the grinding at the lowest point in the middle has the best effect, while the grinding on two edges has the worst effect.

Grinding with a Constant Removal Rate.In the forming process represented by aspherical grinding, when the grinding adopts a constant feeding rate, the removal amount (or envelope contact 
width $L^{\prime}$ ) of grinding wheel at different processing points is different due to the changes in the surface curvature, and the residual height of the workpiece surface is also different, as shown in Fig. 9. The stress size of wheel changes accordingly, causing the different cutter relieving amount, as well as the actual processing path inconsistent with the theoretical processing path ${ }^{8]}$. Meanwhile, the roughness of machined surface also has greater differences. To this end, the Professor Shoji Kexiong of Tohoku University first proposed the idea of achieving the feeding with constant removal amount by controlling the feeding rate of grinding wheel so as to reduce the errors caused by feeding ${ }^{[9,10]}$. According to the equation 1, the relationship between the feeding residual height $\mathrm{Rr}$ and the feeding rate of grinding wheel with curved surface by AEGM can be deduced:

$$
\begin{aligned}
& \frac{\partial \rho}{\partial v_{r}}=\frac{1}{(\rho-r)\left[(\rho-r)^{2}-v_{r}{ }^{2} /\left(4 n_{w}{ }^{2}\right)\right]^{-1 / 2}-1} \cdot\left\{\left[r^{2}-v_{r}{ }^{2} /\left(4 n_{w}{ }^{2}\right)\right]^{-1 / 2} \cdot v_{r}{ }^{2} /\left(4 n_{w}{ }^{2}\right)+\right. \\
& \left.\left[(\rho-r)^{2}-v_{r}{ }^{2} /\left(4 n_{w}{ }^{2}\right)\right]^{-1 / 2}\right\}
\end{aligned} .
$$

From the above equation, $\partial \rho / \partial V_{r}>0$, during the grinding of revolving curved surface, in order to ensure the uniform surface roughness of the entire curved surface and the shape accuracy of the surface, the value of residual height should be kept the same. When the arc radius $r$ of wheel section is constant, and the workpiece speed $n_{w}$ is constant, where the curvature radius increases, the wheel feeding rate $V_{r}$ should also be increased; where the curvature radius decreases, the wheel feeding rate should be minimized, as shown in Fig. 10. According to this formula, after the workpiece is sectionalized and an ideal value of roughness is given, the corresponding feeding rate at the approximately equal radius of curvature can be obtained.

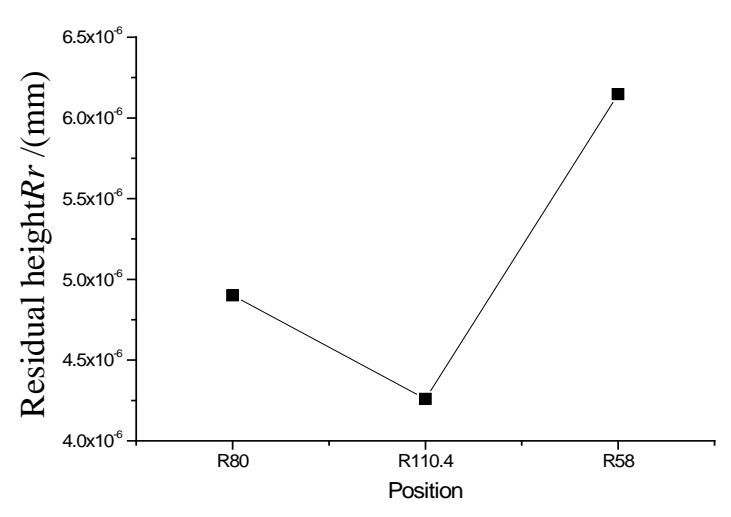

Fig.9 Residual height at different positions

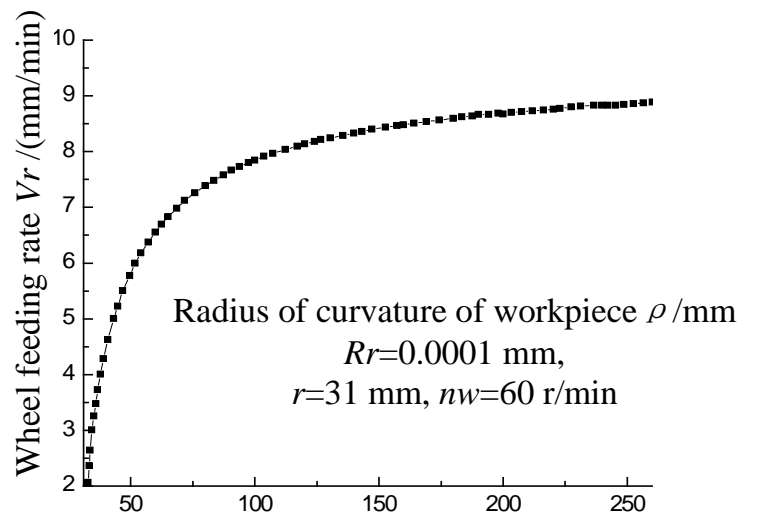

Fig.10 Wheel feeding speed at different positions

From Fig. 9, we can know, in the case of constant feeding rate, if the radius of curvature of the curved surface is R80, R140 and R58 respectively, then the feeding residual height of grinding wheel is $0.0049 \mu \mathrm{m}, 0.0042 \mu \mathrm{m}$, and $0.0061 \mu \mathrm{m}$ accordingly. The volatility of residual height on the entire curved surface is $30.7 \%$. The rate of change of surface roughness after using a constant feeding rate is $35.06 \%$. The surface roughness of each part is R80: Ra0.36 $\mu \mathrm{m}, \mathrm{R} 110.4: 0.32 \mu \mathrm{m}$, and R58: $0.49 \mu \mathrm{m}$, respectively. The change rule is almost the same with the ideal value of residual height. During the arc envelope grinding, the use of variable-speed grinding (as shown in Fig. 10) can effectively reduce the surface roughness. With the change of feeding rate of grinding wheel at different parts of the curved surface, there may be a slight decline in the surface roughness, and the volatility of surface roughness is reduced from $35.06 \%$ to $11.33 \%$,as shown in Fig. 11. 

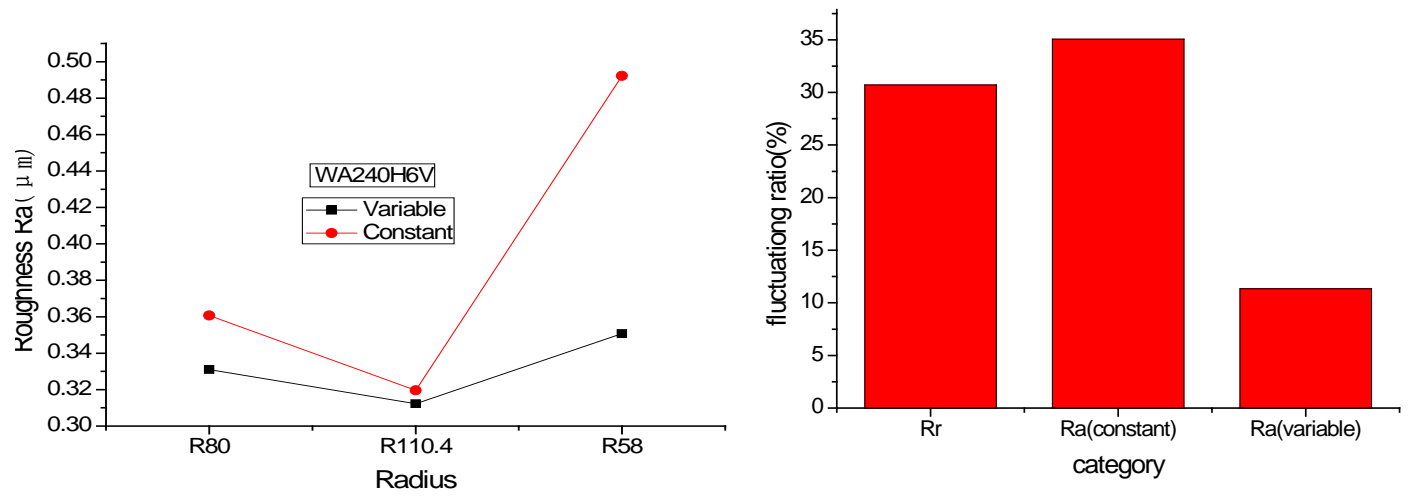

(Grinding ap0.01mm, total grinding thickness of $0.5 \mathrm{~mm}$ )

Fig.11 Wheel feeding rateVs.roughness

\section{Summary}

In this paper, the differences between the grinding of curved surface and ordinary grinding are analyzed, including contact mode, residual height, movement of grinding point, and contact arc length, etc. Based on the analysis of displacement distance of grinding point, the relationship between the grinding arc length, and arc radius of the grinding wheel as well as curvature radius of the workpiece is obtained; meanwhile, the range of arc radius of grinding wheel to prevent interference and incomplete grinding is solved. Through the calculation of contact arc length between the grinding wheel and the workpiece, it can be obtained that the greater the curvature radius of the workpiece is, the smaller the contact arc length and the contact area are. To ensure the consistent shape accuracy and surface roughness of the workpiece, it is proposed to section the workpiece, so the appropriate wheel feeding rate at different radius of curvature can be obtained. After using the sectionalized variable-speed grinding, the volatility of roughness of entire curved surface is reduced to $11.33 \%$.

\section{References}

[1] J.Xie and J.Tamaki: International Journal of Machine Tools \& Manufacture Vol. 48 (2008), p. 1111-1119

[2] J.S. Lee, M. Saeki, and T.Kuriyagawa: International Journal of the Korean Society of Precision Engineering Vol. 4 (2003), p. 48-54

[3] Y.L.Kong, Q. S. Yan and J. H. Song: China Mechanical Engineering Vol. 19 (2008), p. 2557-2560

[4] T. Kuriyagawa, T. Tachibana, K. Syoji and Y. Mori: Transactions of the Japan Society of Mechanical Engineers Vol. 63 (1997), p. 2532-2537

[5] K. Syoji: Grinding Technique (China Machine Press, Beijing 2007)

[6] J. X. Ren, R. K. Kang and X. K. Shi: Grinding Technology of Difficult-to-machine Materials (National Defence Industry Press, Beijing 1999)

[7] T. Kuriyagawa, K. Syoji and H. Ohshita: Journal of Materials Processing Technology Vol. 136 (2003), p. 39-47

[8] Q. Liu, J. D. Yang, C. L. Tian and J. H. Liu: Optics and Precision Engineering Vol. 6 (2006), p. 1048-1051

[9] M. Saeki, T. Kuriyagawa and K. Syoji: Journal of the Japan Society for Precision Engineering Vol. 68 (2002), p. 1067-1071

[10]Y. B. Guo, J. D. Yang and X. C. Liang: China Mechanical Engineering Vol. 11 (2000), p. 415-417 